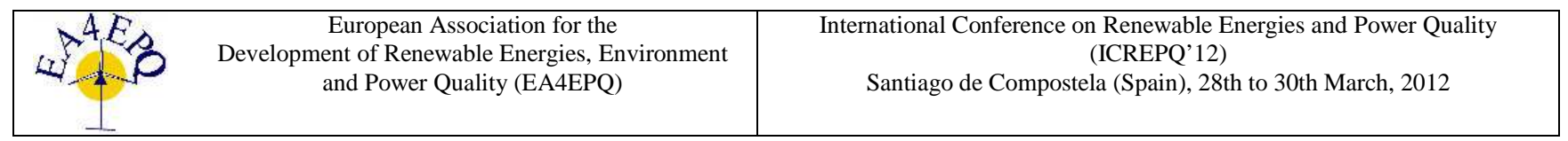

\title{
Complete methodology on generating realistic wind speed profiles based on measurements \\ C. Gavriluta ${ }^{1}$, S. Spataru ${ }^{2}$, I. Mosincat ${ }^{2}$, C. Citro ${ }^{1}$, I. Candela ${ }^{1}$, P. Rodriguez ${ }^{1}$ \\ ${ }^{1}$ Renewable Electrical Energy Systems (SEER), Technical University of Catalonia. \\ Campus of Terrassa, 08222 Barcelona (Spain)
}

Phone/Fax number: +0034 937398291, e-mail: catalin.gavriluta@upc.edu, costantino.citro@upc.edu, candela@ee.upc.edu, prodriguez@ee.upc.edu

${ }^{2}$ PV-MG research group, Aalborg University, Department of Energy Technology, Pontoppidanstraede 101, 9220 Aalborg, (Denmark)

Phone/Fax number: +0045 5264 7520, e-mail: $\underline{\text { ssp@et.aau.dk, imosin09@student.aau.dk }}$

\begin{abstract}
The wind speed represents the main exogenous signal applied to a Wind Energy Conversion System (WECS) and determines its behavior. The erratic variation of the wind speed, highly dependent on the given site and on the atmospheric conditions, makes the wind speed quite difficult to model. Moreover, wind modelling for medium and large time scales is poorly treated in the present literature. This paper presents methods for generating realistic wind speed profiles based on real measurements. The wind speed profile is divided in a lowfrequency component (describing long term variations) and a turbulence component (corresponding to fast, high frequency variations). For modelling the low frequency component two methods are implemented and compared: one based on the Van der Hoven spectrum and one based on auto regressive models. The importance of the turbulence component is also analyzed and modelling methods based on white noise filtering are presented.
\end{abstract}

\section{Key words}

wind speed modelling, medium and large time scales, van der hoven, autoregressive models

\section{Introduction}

A wind turbine converts the wind energy into mechanical energy and then through the generator in electrical energy. The energy contained in the wind depends on the air density, the rotor area and the wind speed.

The density of air influences directly its mass, and therefore the kinetic energy in the wind. In other words, the higher the density, the heavier the air and more energy is received by the turbine.

Secondly, the rotor area, or rotors swept area, determines how much energy a wind turbine is able to produce from the wind. The rotor area increases with the square of the rotor radius, and so is the output power of the turbine. And finally, the most important factor for the total available energy a turbine can convert into electricity is the wind speed. The energy in the wind varies with the cube of the average wind speed. Considering all the above, the power available in the wind is defined by Eq. (1):

$$
P=\frac{1}{2} \rho A V^{3}
$$

where $\mathrm{P}$ is the instantaneous energy (power) in the wind, A is the rotor area, $\rho$ is the air density and $\mathrm{V}$ is the wind speed.

The energy available in the wind is derived from Eq. (1) by integrating the wind speed over a period of time $t p$, typically one year [1], resulting the following:

$$
\text { Total_Energy }=\frac{1}{2} \rho A \int_{0}^{t p} V(t)^{3} d t
$$

It is necessary to highlight that taking the average wind speed (from a Weibull distribution) and then estimating the average energy based on that value could lead to nonrealistic results when computing the total energy using Eq. (2). This is because the contained energy in the wind is considerably larger for high wind speeds.

Therefore the need of accurate wind models, especially in the area of energy storage investigation, can easily be identified.

The erratic variation of the wind speed, highly dependent on the given site and on the atmospheric conditions, makes the wind speed even more difficult to model. A common simplification is to assume thermic equilibrium of the atmosphere nearby Earth (neutral atmosphere [2]). Therefore, turbulence results mainly from the friction between air and ground, due to the ground roughness [3]. Wind near the Earth's surface is characterized in a general sense by a spatial (3D) speed distribution. However, assuming that the turbine is equipped with yawing equipment, and that changes in wind direction are sufficiently slow, then the turbine rotor is maintained normal to the wind direction. Hence, the wind turbine model requires only the longitudinal wind speed to be modelled [3]. Therefore, in the present paper only scalar (1D- fixed-point) wind speed models will be addressed. Another assumption is that the wind turbine runs in 
normal operating regimes, so the developed models will not include extreme operating conditions like wind gusts. With these assumptions in mind, the fixed-point wind speed model has been developed in the literature $[1,2,3$, 4] considering the wind dynamics as a result from combining meteorological conditions with particular features of a given site. Thus, wind speed is modeled by a non-stationary random process, obtained by superposing two components, as seen in Eq. (3):

$$
v(t)=v_{m}(t)+v_{t}(t)
$$

where $v_{m}(t)$ is the low-frequency component (describing long term, low-frequency variations) and $v_{t}(t)$ is the turbulence component (corresponding to fast, high frequency variations) [2].

These components can be identified in Van der Hoven's large band (six decades) model, presented in Fig. 1, developed by Van der Hoven (1957), from long and short term records at Brookhaven, New York, and first published in [5]. The spectral gap of around $0.5 \mathrm{mHz}$ suggests that the turbulence component can be modeled as a zero average random process (there is little energy in the spectral range between $2 \mathrm{~h}$ and $10 \mathrm{~min}$ ).

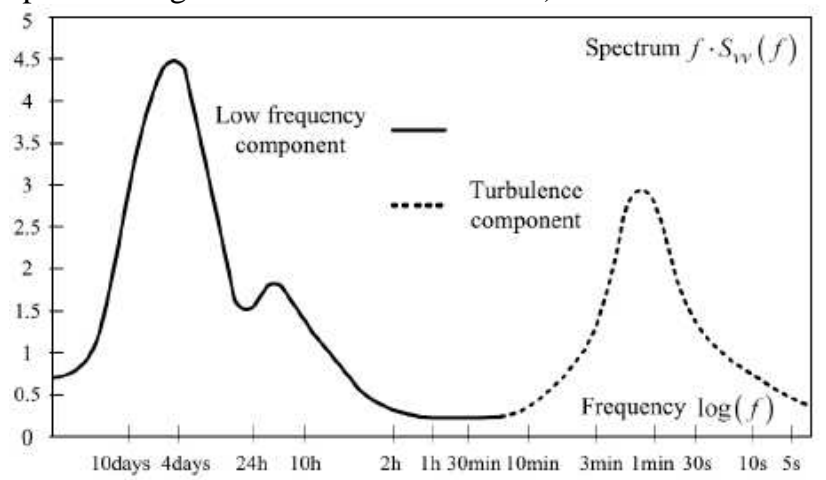

Figure 1. Van der Hoven's spectral model of the wind speed [3]

\section{Modelling of mean wind speed}

The low frequency component of the wind speed, corresponding to the geostrophic winds, is usually referred to as quasi-steady mean wind speed or just mean wind speed. The value of the mean wind speed is obtained as the average of the instantaneous speed over an interval $t_{p}[1]$.

$$
V_{m}=\frac{1}{t_{p}} \int_{t_{0}-\frac{t_{p}}{2}}^{t_{0}+\frac{t_{p}}{2}} V(t) d t
$$

The knowledge of the mean wind speed that can be expected at a potential site is crucial to determine the economic viability of a wind energy project. These data are also essential to select the WECS in order to maximize efficiency and durability [1]. The probability distribution of the mean wind speed is predicted from measurements collected during several years. All these data are usually arranged in a histogram. The wind distribution experimentally obtained can be approximated by a Weibull distribution, such as that shown in Fig. 2. The Weibull distribution is given by Eq. (5).

$$
V_{m}=\frac{\alpha}{\beta^{\alpha}} V_{m}^{\alpha-1} \exp \left(-\frac{1}{\beta^{\alpha}} V_{m}^{\alpha}\right) ; V_{m} \geq 0 ; \alpha, \beta>0
$$

where $\alpha$ and $\beta$ are shape and scale parameters. These parameters are adjusted to match the wind data at a particular site [1]. The Weibull probability function reveals that large mean wind speeds rarely occur whereas moderate winds are more frequent.

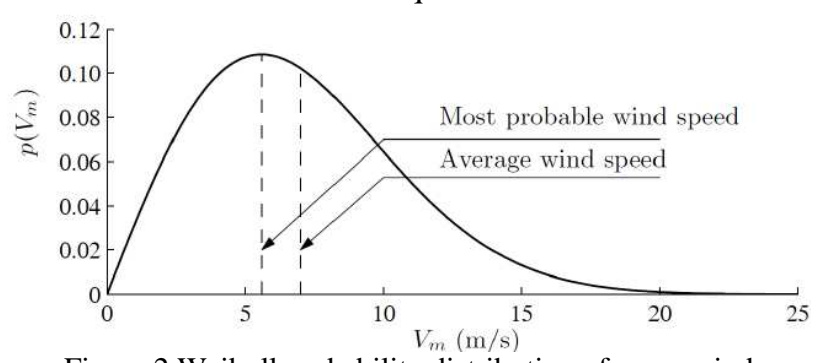

Figure 2.Weibull probability distribution of mean wind speed [1].

In the particular case of Fig. 2, the most probable mean wind speed is approximately $5.5 \mathrm{~m} / \mathrm{s}$ whereas the average wind speed is $7 \mathrm{~m} / \mathrm{s}$.

As was mentioned in the previous section, the averaging period is chosen to lie within the energy gap, more common around 10 to 20 minutes. When this is the case, the macro meteorological changes in wind speed appear as slow fluctuations of the mean wind speed [1].

Starting from these presumptions, a wide variety of methods for generating and forecasting wind time series based on wind speed measurements have been developed $[6,7]$ in the literature, Weibull distribution method, spectrum methods, autoregressive methods, Markov chain methods, wavelet decomposition or neural networks based methods.

In the following two of most commonly employed methods for generating wind speed time series will be presented and analyzed.

\section{A. Van der Hoven spectrum method}

Maybe the most common and simple method for generating accurate and persistent wind speed time series is by sampling the Van der Hoven spectrum. The idea, presented in [4], is quite simple and intuitive. Starting from the Van der Hoven spectrum, prior calculated for the desired time span and sampling accuracy (usually 10 minutes to 1 hour samples), from the available measurement data, the spectrum is sampled in order to obtain a new wind speed time series.

More specifically, the frequency range below $1 / t_{p}$ is divided into $\mathrm{m}$ intervals. Then, the mean wind speed is computed at every step as:

$$
V_{m}(t)=V_{0}+\sum_{t=1}^{m} A_{i} \cos \left(\omega_{i} t+\xi_{i}\right)
$$

where $\omega_{i}=\overline{1, m+1}$; is the discretized angular frequency, $\xi_{i}$ is a stochastic variable uniformly distributed in $[-\pi, \pi][4], V_{0}$ is the mean speed measured during a period much longer than $2 \pi / \omega_{i}$ (the largest period in Van der Hoven's characteristic) and $A_{i}=\frac{2}{\pi} \sqrt{\frac{1}{2}\left(S_{v v}\left(\omega_{i}\right)+S_{v v}\left(\omega_{i+1}\right)\right)\left(\omega_{i+1}-\omega_{i}\right)}$ with $S_{v v}\left(\omega_{i}\right)$ being the power spectral density at $\omega_{i}[4]$. 
One important advantage of this method, besides its simplicity, is that the generated wind speed time series has a similar Van der Hoven spectrum in the low frequency side as the original measurement data, preserving periodical trends. Moreover the generated data is persistent.

Fig. 3, Fig. 4 and Fig. 5 show the results obtained by applying this method and using 10 minutes wind speed averages. The 10 minutes average data has been recorded during an entire year at the Capel wind site, UK (courtesy of www.winddata.com).

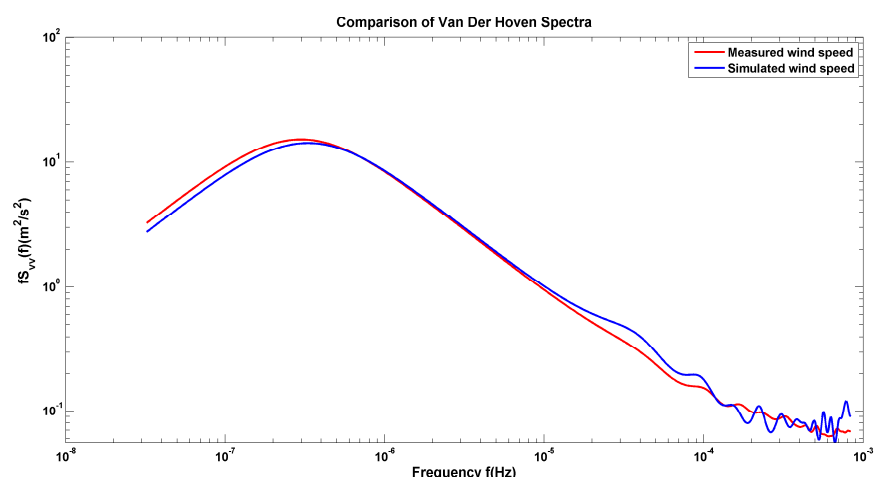

Figure 3. Comparison between the Van Der Hoven Spectra of the measured data (red) and data generated using the Van der Hoven spectrum method (blue).
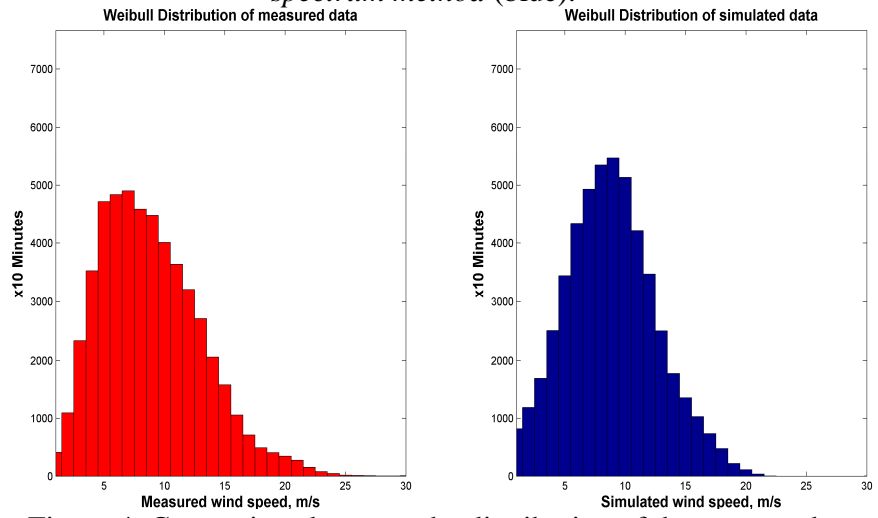

Figure 4. Comparison between the distribution of the measured data (red) and data generated using the Van der Hoven spectrum method (blue).
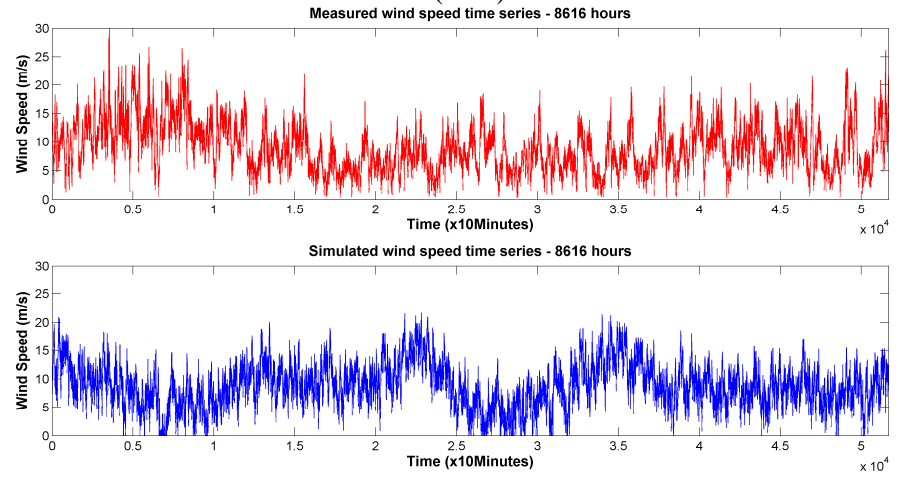

Figure 5. Comparison between the time evolution of the measured data (red) and data generated using the Van der Hoven spectrum method (blue).

\section{B. Autoregressive method}

The use of autoregressive models for generating hourly mean wind speed time series is reported in the literature very commonly $[6,7,8]$. The reason for this widespread use is because of the high dependence of hourly mean wind speed time series [6]. This property particularly requires a wind speed data generation model incorporating the dependence structure of the observations. Weibull distributed random numbers (another simple method of emulating wind speed) do not take this property into account as they are independent, but autoregressive models are of correlated type and hence capable of simulating this property of the wind speed data series [6].

Two of the most often employed auto regressive models used are the p-th order autoregressive model, presented in Eq. (7), and the p; q-th order autoregressive moving average models, presented in Eq. (8), also known as Box-Jenkins models $[6,7]$.

$$
\begin{gathered}
A R(p): y_{i}=\sum_{j=1}^{p} \phi_{j} y_{i-j}+\varepsilon_{i} \\
\operatorname{ARMA}(p, q): y_{i}=\sum_{j=1}^{p} \phi_{j} y_{i-j}+\sum_{k=1}^{q} \theta_{k} e_{i-k}+\varepsilon_{i}
\end{gathered}
$$

where $\phi_{j}$ are the autoregressive, $\theta_{k}$ the moving average parameters and $\varepsilon_{i}$ is a random component of normal distribution, with zero mean and unity variance.

These two models were fitted in [8] and [9], yielding very good results. The methodology used in both papers is similar, and takes into account the non-Gaussian distribution, the diurnal non-stationarity, and the autocorrelated nature of wind speed [8].

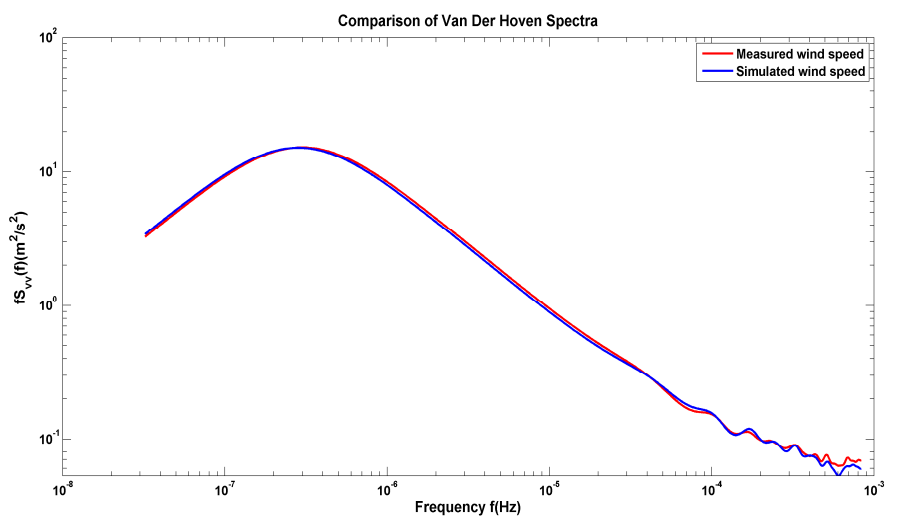

Figure 6. Comparison between the Van Der Hoven Spectra of the measured data (red) and data generated using the Autoregressive method (blue).

The advantages of this method, although complex, are numerous, and take into account several characteristics of the wind speed, namely auto-correlation, non-Gaussian distribution and diurnal non-stationarity. And since no assumptions or previously estimated factors are introduced in the models, this approach ensures that there is no inherent distortion in the resulting model [9].

Time series models of wind speed developed using this methodology have many potential uses, including simulation and short period forecasting of wind speed and at a single site [8]. 

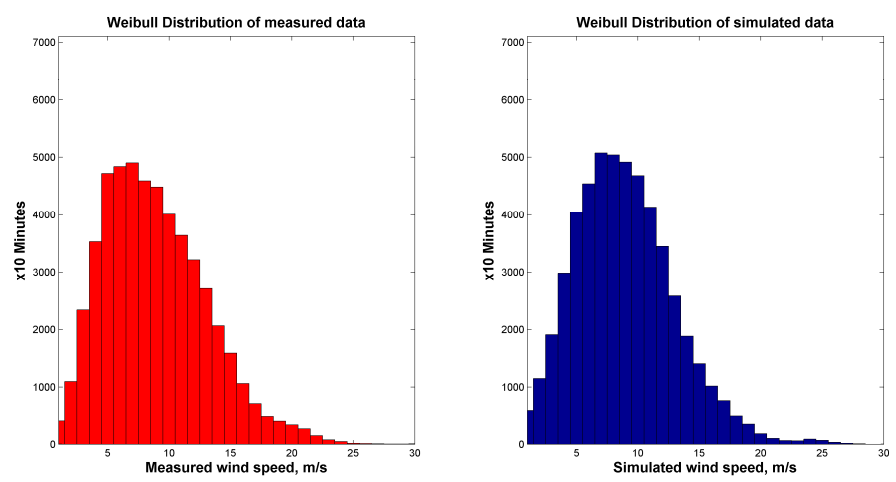

Figure 7. Comparison between the distribution of the measured data (red) and data generated using the Autoregressive method (blue).
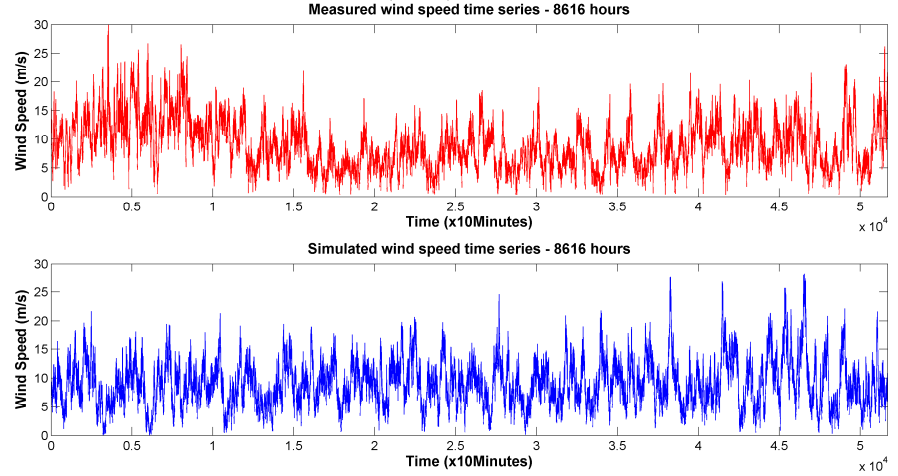

Figure 8. Comparison between the time evolution of the measured data (red) and data generated using the Autoregressive method (blue).

Fig. 6, Fig. 7, and Fig. 8 present the results obtained by this method using as a starting point the same measured data as in the previous case.

It can be observed that the autoregressive methods are superior to the spectrum methods, providing results that approximate better the statistical characteristics of the real data.

\section{Modelling of local effects}

As mentioned earlier, the wind speed at a fixed point can be divided in two components: the mean speed (determined as 10 or 20 minutes averages) and the turbulence. Section 2 has presented methods for mathematically describing the wind in terms of frequencies above the energy gap in the Van der Hoven spectrum.

In order to model the fast changing components in the wind also known as turbulence and the local effects, one has to start by looking at the shape of the Van der Hoven spectrum at high frequencies.

The common approach used in the literature in order to model wind turbulence is to approximate the wind spectrum at high frequencies with the Kaimal or the von Karman spectrum. According to [1] the following models can be adopted for the two spectrums:

$\Phi(\omega)=\frac{K_{v}}{\left(1+\omega \cdot T_{v}\right)^{5 / 3}}$, Kaimal Spectrum

$\Phi(\omega)=\frac{K_{v}}{\left(1+\left(\omega \cdot T_{v}\right)^{2}\right)^{5 / 6}}$, von Karman Spectrum

Both models are parameterized by $\mathrm{K}_{\mathrm{v}}$ and $\mathrm{T}_{\mathrm{v}}$ and according to [1] they are dependent on the mean wind speed and parameters of the site, like the turbulence length scale and the turbulence intensity.

It is difficult to say which of the two models is more suited for turbulence modelling. Even though the frequency response of the two does not present any major differences, Kaimal is widely accepted and suggested by the Danish standard for loads and safety of wind turbines construction.

Common practice, presented in [4] as well as in [10], used in order to obtain the desired shape of the spectrum in the high frequency area is to pass white noise through a shaping filter tuned based on the von Karman or the Kaimal spectrum.

The methods presented so far for modelling the mean wind speed together with the turbulence offer a clear overview of the wind speed at a fixed point for time scales ranging from seconds to years. However, placing a wind turbine in the wind flow generates extra turbulences, mainly because of the tower shadow and the wind shear. The distribution of wind is altered by the presence of the turbine tower. According to [10] the wind speed in the front of the tower can be reduced with up to $20 \%$, the tower having an averaging low-pass-filter effect on the wind speed. Also, the rotating blades experience different wind speeds according to their position; for example a blade pointing upwards will experience higher wind speeds than when pointing downwards because wind speed varies with height as stated by the Prandtl logarithmic law.

Not taking these aspects into consideration when looking at power fluctuations analysis will yield non-realistic results. In order to make the prediction of the wind speed experienced by a wind turbine as accurate as possible, models that take into account wind shear and tower shadow were used for modelling the turbulence component.

There are at least three methods for modelling the local effects of the wind speed. In this paper focus was set on the harmonics filter method from [11, 12] and on the spatial/rotational filter method from [3].

The harmonics filter method, implemented in [12], uses the Kaimal spectrum for modelling the turbulence, and admittance filters for covering the effects of wind speed averaging and rotational sampling effects. The proposed structure can be better pictured by analyzing Fig. 9 .

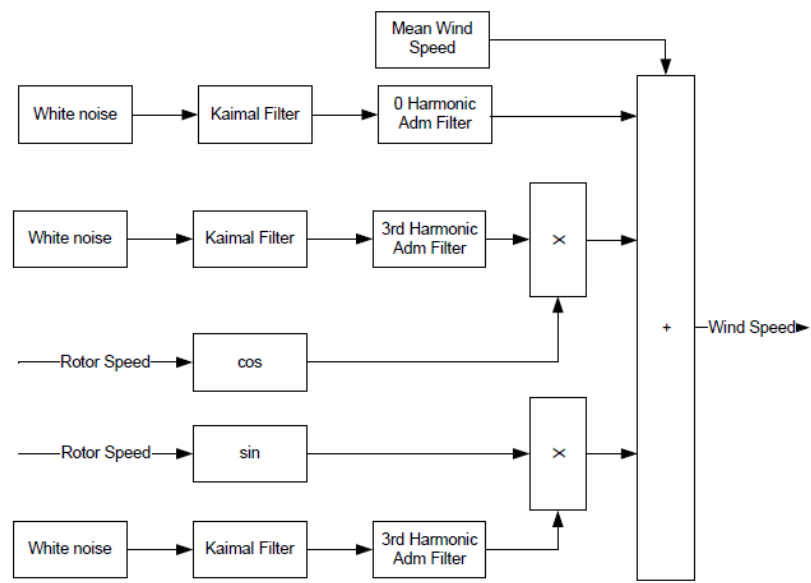

Figure 9. Harmonics filter method. Block Diagram. 
The problem that arises is related with the tuning of the three filters: Kaimal, Zero Harmonic Admittance Filter and Third Harmonic Admittance Filter.

Firstly the Kaimal filter will be described. Extensive work on this topic has been done in [11], where rational filters of different orders have been fitted to experimental data, in order to provide an approximation as good as possible to the initial shape of the Kaimal filter spectrum. For the work presented in this paper, the usage of a second order filter has been chosen. The work presented in [12] uses the same filter, and by analyzing the data provided by [11] it can be seen that it provides a good compromise between complexity and accuracy. The transfer function of the filter is the one presented in Eq. (11), where $\mathrm{c}=\mathrm{V}_{\mathrm{m}} / \mathrm{Lt}$ and $\mathrm{K}=$ $V_{m} / \sqrt{2 L_{t} \sigma^{2}}$, with $\mathrm{L}_{\mathrm{t}}$ being the turbulence length scale, $\mathrm{V}_{\mathrm{m}}$ the mean wind speed and $\sigma$ the turbulence standard deviation. Both $\mathrm{L}_{\mathrm{t}}$ and $\sigma$ are parameters related to the location.

$H_{\text {Kaimal }}(s)=K \frac{0.0182 c^{2} \cdot s^{2}+1.3653 c \cdot s+0.9846}{1.3463 c^{2} \cdot s^{2}+3.7593 c \cdot s+1}$

The next question is related to the two admittance filters, and the answer was found in [11]. Once again rational filters of different orders were fitted to measured data, and the results for second order filters are presented in the Eq. (12) and Eq. (13) where $d=R / V_{m}$ with $R$ being the rotor radius and $\mathrm{V}_{\mathrm{m}}$ the mean wind speed.

$$
\begin{aligned}
& H_{a d m 0}(s)=\frac{4.7869 d^{2} \cdot s^{2}+0.9904}{7.6823 d^{2} \cdot s^{2}+7.3518 d \cdot s+1} \\
& H_{a d m 3}(s)=\frac{0.2766 d^{2} \cdot s^{2}+0.0307}{0.3691 d^{2} \cdot s^{2}+1.7722 d \cdot s+1}
\end{aligned}
$$

The idea behind this method is to produce a single equivalent wind speed, which is further used as input to the aerodynamic model. Some aspects are taken into consideration while developing this model. Firstly is that the wind model contains only the zero and the third harmonic due to the fact that the three bladed rotor is perfectly symmetrical [13]. Another aspect that is considered is that the wind turbine structure acts like a low pass filter [13], averaging the wind speed.

In order to achieve the desired output, firstly the zero and third harmonic of the fixed point turbulence are generated using shaping filters tuned based on the Kaimal spectrum. Then two admittance filters, fitted in [11], are applied to the two harmonics. The one applied to the zero harmonic is tuned so that it simulates the averaging effect, while the one applied to the third one, takes into account the variations due to the rotational turbulence and tower shadow in the wind speed field over the rotor disk.

The two filtered harmonics are added in the end in order to obtain the equivalent wind speed.

The second model, proposed in [3], takes a similar approach as the previous one. Two filters are introduced after the fixed point wind speed: one that takes care of the averaging effect (the Spatial Filter), and one that takes into account the effect of the shear, the tower shadow and other effects introduced by the rotating blades (the Rotational Sampling Filter). The way these filters are interconnected with the fixed point wind speed is different from the previous method, hence the new structure can be found in Fig. 10. Another difference that can be noticed is that for the generation of the fixed point speed a von Karman filter is used this time. The transfer function

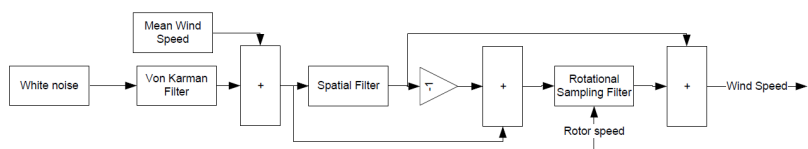

Figure. 10 Spatial/rotational filter method. Block Diagram.
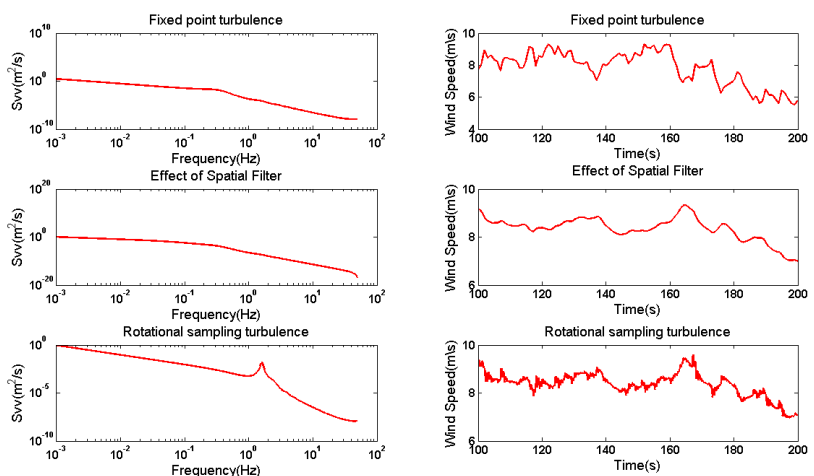

Figure 11. Effect of the spatial and rotational filter on the spectrum and time characteristic

chosen for this filter is the one proposed by [4], here presented in Eq. (14) where $\mathrm{T}_{\mathrm{F}}=\mathrm{L}_{\mathrm{t}} / \mathrm{V}_{\mathrm{m}}$ and $\mathrm{K}_{\mathrm{F}}=\sqrt{\frac{2 \pi}{B(1 / 2,1 / 3)} \cdot \frac{T_{F}}{T_{s}}}$ with $\mathrm{B}(\mathrm{x} ; \mathrm{y})$ being the beta function and Ts the sampling time.

$$
H_{\text {Karman }}(s)=K_{F} \frac{0.4 \cdot T_{F} \cdot s+1}{\left(T_{F} \cdot s+1\right)\left(0.25 T_{F} \cdot s+1\right)}
$$

For the spatial filter the transfer function is presented in Eq. (15), where $a_{s f}=0 \ldots 55$ is an empirical factor, and $b_{s f}$ is a parameter describing the correlation between wind speed in different points across the rotor; $b_{\text {sf }}$ equals 1.3. $\left(\mathrm{R} / \mathrm{V}_{\mathrm{m}}\right)$, where $\mathrm{R}$ is the blade length and $\mathrm{V}_{\mathrm{m}}$ the average wind speed experienced by the hub.

$$
\begin{aligned}
& H_{s f}(s)=\frac{\sqrt{2}+b_{s f} \cdot s}{\left(\sqrt{2}+b_{s f} \cdot \sqrt{a_{s f}} \cdot s\right)\left(1+\frac{b_{s f}}{\sqrt{a_{s f}}} s\right)} \\
& H_{r s}(s)=\frac{\left(s+N_{b} \cdot \Omega_{l}+\varepsilon\right) \cdot\left(s-N_{b} \cdot \Omega_{l}-\varepsilon\right)}{(s+\sigma)^{2}+\left(N_{b} \cdot \Omega_{l}\right)^{2}}
\end{aligned}
$$

Equation (16) presents the transfer function proposed in [3] for the Rotational Sampling Filter. In the equation $N_{b}$ represents the number of rotor blades, $\Omega_{l}$ the rotational speed of the rotor, while $\varepsilon$ and $\sigma$ are two parameters that determine the magnitude of the power density concentration and the characteristic's selectivity at the $\mathrm{N}_{\mathrm{b}} \cdot \Omega_{l}$ frequency [3]. Direct indications on how to choose $\varepsilon$ and $\sigma$ are not provided in the previously cited work, so after some tuning the following values were selected as default values: $\varepsilon=0$ and $\sigma=1$ for the work presented here.

The two presented methods provide similar results in modelling the fast components of the wind speed. As emphasized before, it is very important to take into consideration the effects produced by the interaction between the wind and the turbine when dealing with problems related to power estimation. Figure 11 presents the spectrum and the time series for three cases: the top 
one represents the fixed point wind speed, the one in the middle emphasizes the averaging effect of the spatial filter, while the graphs in the bottom of the figure show the effect of the rotational sampling filter. Clear differences can be spotted in the spectrum as well as in the time series. Ignoring these differences and considering just the fixed point wind speed could over-estimate the wind resource at a certain location.

\section{Conclusion}

Reliable and consistent wind speed testing data is imperative for the realistic study and validation of a wind energy conversion system and consequently of an energy storage system. Since various wind speed scenarios need to be developed with various time scales and wind site characteristics, and appropriate wind speed measurements are not always readily available, two methods for generating synthetic mean wind speed have been implemented and compared. These two methods were chosen from among a broad range of available and currently developing methods for generating and forecasting mean wind speeds, because of their good agreement on ease of use and accuracy of results.

The next important aspect that has to be taken into consideration when generating synthetic wind speed is the high frequency components (turbulence), below 10 minutes time periods, which are not included in the mean wind speed, but have a significant impact on short period variations of the WECS's power output. This phenomenon, as mentioned before, is of great importance to energy storage and has been studied in detail. Two approaches for dealing with these site specific local effects have found consensus in the research community, and have consequently been implemented and compared.

\section{Acknowledgment}

This work was supported in part by the projects ENE201129041-C02-01 /ALT and TRA2009-01-03 of Spanish Ministry of Science and Innovation.

\section{References}

[1] Fernando D. Bianchi, Hernn De Battista, and Ricardo J. Mantz. Wind Turbine Control Systems. Principles, Modelling and Gain Scheduling Design. Springer, London, 2007.

[2] Tony Burton, David Sharpe, Nick Jenkins, and Ervin Bossanyi. Wind energy handbook. Wiley, New-York, 2001.

[3] Iulian Munteanu, Antoneta Iuliana Bratcu, Nicolaos-Antonio Cutululis, and Emil Ceanga. Optimal Control of Wind Energy Systems: Towards a Global Approach. Springer Science+Business Media Deutschland GmbH, 2008.

[4] C. Nichita, D. Luca, B. Dakyo, and E. Ceanga. Large band simulation of the wind speed for real time wind turbine simulators. IEEE Transactions on Energy Conversion, 2002.

[5] Isaac Van der Hoven. Power spectrum of horizontal wind speed in the frequency range from 0.0007 to 900 cycles per hour. Journal of Meteorology, 1957.

[6] H. Aksoy, Z. F. Toprak, A. Aytek, and N. E. nal. Stochastic generation of hourly mean wind speed data. Renewable Energy, 2004.

[7] A. Sfetsos. A comparison of various forecasting techniques applied to mean hourly wind speed time series. Renewable Energy, 2000.
[8] R. Billinton, H. Chen, and R. Ghajar. Time-series models for reliability evaluation of power systems including wind energy. Microelectronics Reliability, 1996.

[9] Barbara G. Brown, Richard W. Katz, and Allan H. Murphy. Time series models to simulate and forecast wind speed and wind power. Journal of climate and applied meteorology.

[10] Pedro Rosas. Dynamic Influences of Wind Power on the Power System. PhD thesis, Denmark Technical University, March 2003.

[11] Wiebke Lagrander. Models for variable speed wind turbine. Master's thesis, CREST Loughborough University UK, 1996.

[12] Poul Sorensen, Frede Blaabjerg Florin Iov, Anca Daniela Hansen. Wind turbine blockset in Matlab/simulink. Technical report, Aalborg University, 2004.

[13] Frede Blaabjerg Anca D. Hansen, Poul Srensen and John Becho. Dynamic modelling of wind farm grid interaction. 